\title{
An Important Pitfall in Diagnosing Intracanalicular Vestibular Schwannoma
}

$\mathbf{T}$ echnical advances in MR imaging enable scanning of millimeter- and submillimeter-thick slices in a relatively short acquisition time, so sequences such as contrast-enhanced 3D T1 (eg, MPRAGE) are included in standard MR imaging protocols in many institutions. The MPRAGE sequence is especially important for the detection of small lesions such as a small intracanalicular vestibular schwannoma (Koos grade I).

The members of our interdisciplinary schwannoma board have encountered several patients with varying degrees of enhancement in the fundus of the internal auditory canal (IAC) who were referred to us with suspected schwannomas (Fig $1 A-D$ ). However, when we reviewed the patients' initial MR images and the follow-up images, that was a false-positive finding.

Enhancement in the fundus of the IAC is sometimes detected in subjects without pathologic conditions, and it can be unilateral or bilateral and present in varying degrees (Fig $1 A,-C$ ). This is most likely due to the enhancement of vascular structures such as the venous plexus surrounding the nerve sheath (eg, around the Scarpa ganglion) in the acoustic facial cistern ${ }^{1}$ or due to the presence of capillaries in the meningeal layers covering the fundus.

In a recently published study performed using synchrotron phase-contrast imaging, Mei et $\mathrm{al}^{2}$ demonstrated the presence of previously unknown pillars or villi that support the cranial nerves in the IAC and are occasionally associated with blood vessels, mostly capillaries. These vascular structures within the villi may play a key role in the enhancement in this region, and variation

http://dx.doi.org/10.3174/ajnr.A6192 in the amount of blood vessels between different subjects may be one cause for the different degrees of enhancement observed on postcontrast MR images (Fig $1 A,-C$ ).

Therefore, on the basis of our experience, the presence of enhancement in this region should be always correlated with the CISS sequence to confirm or exclude the presence of a nodular lesion, which represents the schwannoma (Fig $1 F$ ).

Knowledge of this pitfall is important so that CISS can be added if such an incidental finding is present, to reduce the number of false-positive cases and thereby reduce the unnecessary follow-up scans and consequentially to lower health care costs. This precaution will also avoid instilling unnecessary fear in patients who incorrectly believe they have a tumor that may affect their hearing and lifestyle.

Disclosures: Arsany Hakim-UNRELATED: Grants/Grants Pending: Swiss Heart Association.* Franca Wagner-UNRELATED: Grants/Grants Pending: Swiss MS Society. * Money paid to the institution.

\section{REFERENCES}

1. Lescanne E, Velut S, Lefrancq T, et al. The internal acoustic meatus and its meningeal layers: a microanatomical study. J Neurosurg 2002;97:1191-97 CrossRef Medline

2. Mei X, Schart-Morén N, Li H, et al. Three-dimensional imaging of the human internal acoustic canal and arachnoid cistern: a synchrotron study with clinical implications. J Anat 2019;234:316-26 CrossRef Medline

(D) Hakim

(iD) Wagner University Institute of Diagnostic and Interventional Neuroradiology Bern University Hospital, Inselspital University of Bern, Bern, Switzerland 

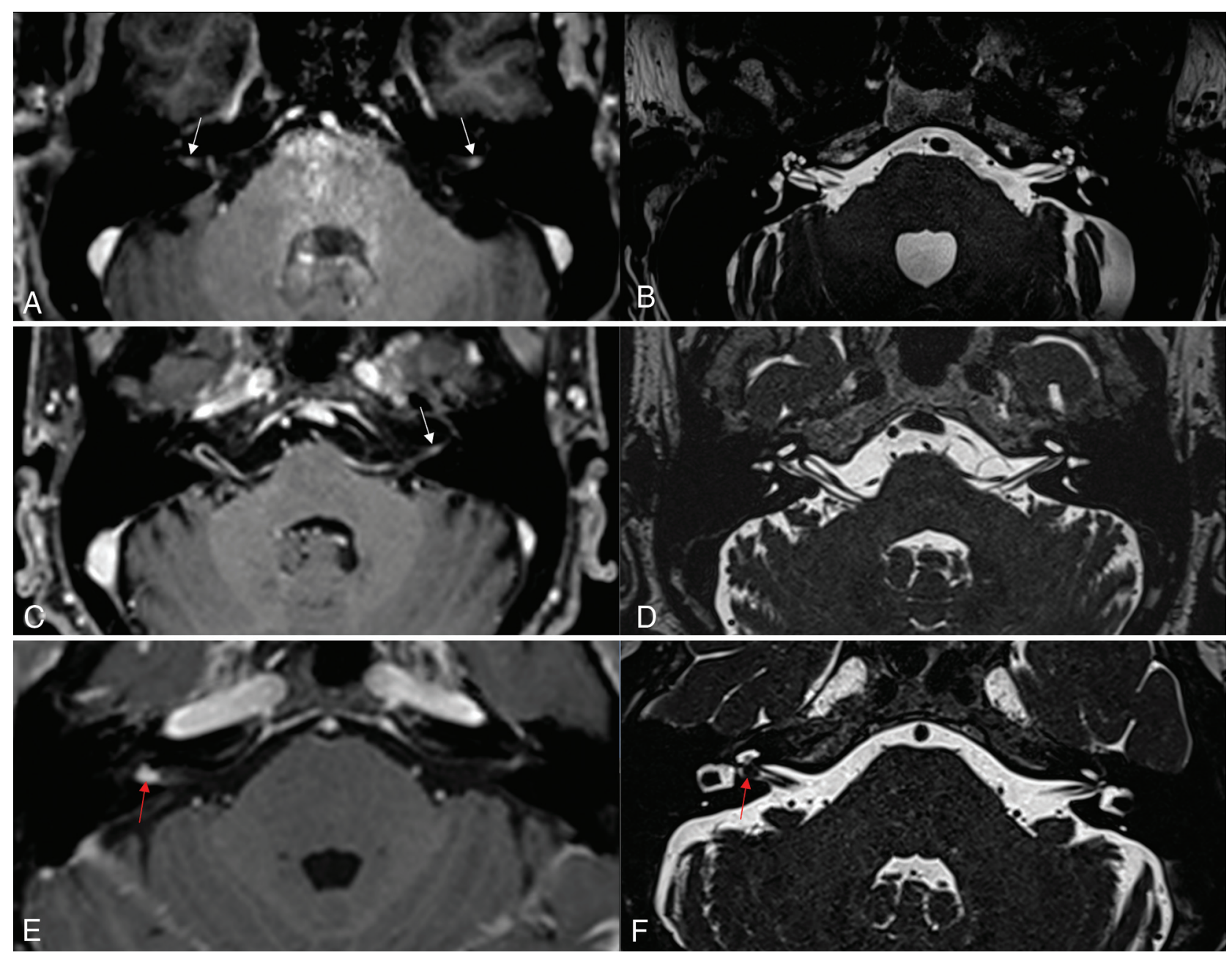

FIG 1. MPRAGE $(A, C$, and $E)$ and $C I S S(B, D$, and $F)$ in 3 different patients (patient $1: A$ and $B$; patient $2: C$ and $D$; and patient 3 : $E$ and $F)$. MPRAGE shows different degrees of enhancement in the fundus of the internal auditory canal bilaterally (left more than right) in patient 1 and on the left side in patient 2 (white arrows in $A$ and $C$ ). However, in both patients, no analogous lesion was detected in the CISS sequence. Therefore, the enhancement was not due to the presence of a schwannoma but was most likely due to a vascular structure. In contrast, in patient 3 , there was nodular enhancement in the fundus of the internal auditory canal on the right side (red arrow in $E$ ), which was accompanied by a corresponding lesion "filling defect" on the CISS sequence (red arrow in F) due to the presence of a small intracanalicular vestibular schwannoma (Koos I). Correlating findings in MPRAGE with the CISS sequence is crucial to avoiding misinterpretation of this enhancement. 